\title{
GENERATING GIS DATABASE OF STREET TREES USING MOBILE LIDAR DATA
}

\author{
M. Yadav ${ }^{1, *}$, P. Khan ${ }^{1}$, A. K. Singh ${ }^{2,1}$, Bharat Lohani ${ }^{3}$ \\ ${ }^{1}$ GIS Cell, Motilal Nehru National Institute of Technology Allahabad, India - ssmyadav@mnnit.ac.in, pkhan773@gmail.com \\ ${ }^{2}$ Dept. of Civil Engineering, Motilal Nehru National Institute of Technology Allahabad, India - aksingh@mnnit.ac.in \\ ${ }^{3}$ Dept. of Civil Engineering, Indian Institute of Technology, Kanpur, India - blohani@iitk.ac.in
}

Commission V, SS: Natural Resources Management

KEYWORDS: Mobile LiDAR, Point Cloud, Street Tree, Tree Crown, Cylinder

\begin{abstract}
:
Mobile LiDAR captures complete details of street trees located along roadway and it is most efficient tool for executing large scale projects for road side trees mapping. Information of road-side trees locations and their morphological characteristics are essential for road widening project planning, road safety and autonomous vehicles. We propose a method to generate GIS database of street trees using information of automatically extracted street trees using mobile LiDAR data. Proposed method first organizes mobile LiDAR input data in partially overlapping cylinders and then morphological characteristics of tree crown and trunk, respectively are used to detect trees falling within cylinders. Finally complete tree structure point cloud is recovered from cylinder by vertical cylinders fitting to crown and trunk separately. The proposed method is tested on point cloud data acquired by StreetMapper 360 of $57 \mathrm{~m}$ long roadway. Completeness and correctness of $88.24 \%$ and $93.75 \%$ respectively are obtained in test site.
\end{abstract}

\section{INTRODUCTION}

As an important component of urban vegetation, street trees play an important role in maintenance of environmental quality, aesthetic beauty of urban landscape, and social service for inhabitants. It lowers road side soil erosion; hence dust level on roads is reduced. Driver perception of roadway edge is improved by road curb side street trees, which improves road safety. Monitoring the changes in morphological conditions of street trees is essential over period for of time for maintaining road safety because sometime trees and their locations become hazardous for roadway vehicles. Road widening projects planning requires detailed information of road-side trees including their locations from existing road edges, density, morphological characteristics to minimize the loss of trees and their cutting cost. So, periodical monitoring, maintenance and management of street trees are important by measuring their locations and morphological parameters. Road side safety needs periodical monitoring of road-side trees size, location and density.

Street trees are generally mapped in tradition way using Global Positioning Systems (GPS) technology and GIS database is generated using location information obtained from GPS. Since last decades, identification of trees and estimation of their morphological parameters are being done using high density airborne LiDAR data (Hyyppä et al., 2008) but at cost of missing details at trunk and lower crown level, respectively. Complete morphological details, i.e., trunk shape and size, lower crown spread of street trees are missing in airborne LiDAR data, which limits its uses for street trees mapping and their morphological parameters estimation.

Advent of mobile LiDAR system (MLS) and its superiority over traditional mapping techniques, including airborne LiDAR accelerate its uses specifically for highway applications (Yadav et al., 2017). MLS collect accurate and dense roadway 3D georeferenced point cloud at road speed without disturbing normal traffic (Yadav et al., 2014). Integration of mobile LiDAR data of roadway and GIS offer a comprehensive way to monitor the street trees. MLS captures every detail of trees along roadway and its mapping speed offer time efficient completion of large scale projects for mapping of road side trees along hundreds of km roadway. MLS is latest, accurate and most efficient tool for measuring complete detail of roadway tree cover. Very limited studies are available in literature for street trees detection using mobile LiDAR data. Mobile LiDAR data were used by researchers to detect street trees by (i) analysing connected components formed by high roughness and low point density ratio (Rutzinger et al., 2010), (ii) Automatic data segmentation and feature extraction using geo-referenced feature image of the point clouds (Yang et al., 2012), (iii) neighborhood searching and marking (Wu et al. 2013), (iv) removing ground points and then off-ground point clustering and segmentation ( $\mathrm{Yu}$ et al., 2014), (v) object matching and retrieval using pairwise 3D shape context (Yu et al., 2014), (vi) first point cloud normalization, then trunk detection and DBH estimation,

* Corresponding author 
and finally crown segmentation (Tao et al., 2015), and (vii) vertical cylinder fitting of different radii using principal component analysis, linearity and orientation of vertical objects (Yadav et al., 2015a). Most of the existing methods detect trees in combination with other pole-like objects like utility pole, traffic lights and signs, bare pole. They are also affected by occlusions and other objects situated close to trees, like buildings, pedestrians, and low vegetation (Yadav and Singh, 2018). In case of connected trees at crown, many methods fail to detect such trees because their methods are clustering based and they form same cluster for all connected trees.

In this paper, we propose a tree extraction method and further generation of GIS database of tree using extracted trees information. The proposed method first organizes data followed by crown and trunk detection. Finally top to bottom fitting is performed to recover complete tree. Proposed method is independent on structuring pattern of input MLS data, pattern of trees and their connectivity unlike existing methods. Proposed method is tested on MLS data of $57 \mathrm{~m}$ long roadway, where trees crowns are connected together and trees trunks are occluded by low vegetation unlike dataset used in literature.

\section{METHODOLOGY}

Proposed method consists of 2 steps, (1) data organization into connected partially overlapping cylinders (2) tree detection and generation of GIS database. In step 1, input 3D point cloud is divided into connected partially overlapping cylinders. Horizontal cross section (disc segment 1) of specific vertical length at tree crown level is chosen from each cylinder. Compactness and area of points within disc segment 1 is computed. Tree crowns within disc segment 1 are detected based on thresholds on compactness and area. Once tree crowns are identified, another horizontal cross section (disc segment 2) of same cylinder at tree trunk level is chosen. Disc segment 2 having same centre and vertical length but small radius compare to disc segment 1. Again criteria on thresholds of compactness and area of disc segment 2 points are checked. In this way complete tree structure is recovered by top to down cylinder fitting using two cylinders of different radii (see Figure 1(b)).

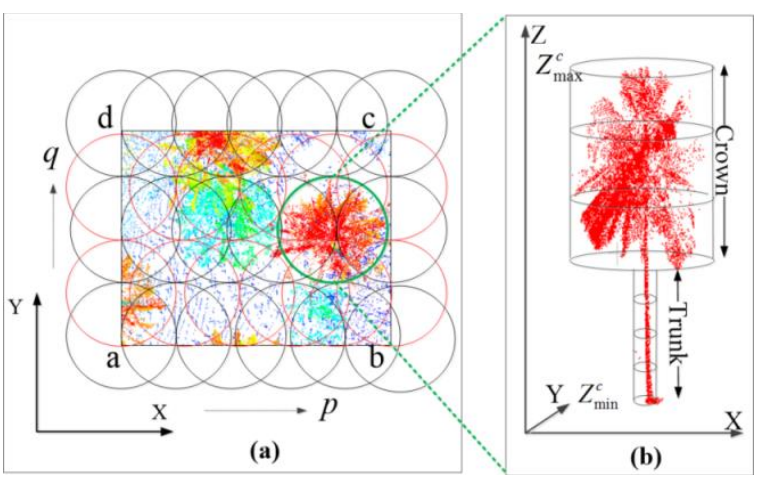

Figure 1. (a) Cylindrical region growing (b) tree detection.
Radius of cylinders at crown and trunk portions, respectively are equal to maximum tree crown and trunk radius, respectively in the dataset. Minimum and maximum $\mathrm{Z}$ values of points lie within cylinder that belong to tree are used to compute tree height. In this way GIS database containing 2D location and height of trees, respectively is generated.

\subsection{Data organization}

Input LiDAR data is first organized into partially overlapping cylinders using method of Yadav et al. (2015a $\&$ b). 3D point cloud is projected on $2 \mathrm{D}$ plane, i.e., $\mathrm{XY}$, and minimum bounding rectangle (MBR), say abcd (see Figure $1(\mathrm{a})$ ) is formed. Vertex a of MBR is chosen initially and circle of radius $R_{\text {crown }}^{\max }$ is drawn. $2 \mathrm{D}$ points lie within circle are recovered using two dimensional k-d tree neighborhood search technique. Next, new circle is formed by changing the centre. Circle centres are updated by adding $\mathrm{R}_{\text {crown }}^{\max }$ in both $\mathrm{X}$ and $\mathrm{Y}$ dimension. In this way $2 \mathrm{D}$ projected data is organized into partially overlapping circles. These circles take shape of cylinders in 3D point cloud. Data organization is used to minimize the effect of features complexities and terrain variations. It is seen through several preliminary experiments. Now input data is composed of partially overlapping cylinders (see Figure 1 (b)) of different height but same circular base. $R_{\text {crown }}^{\max }$ of cylinder is chosen equal to maximum crown diameter of tree available in input data.

\section{$2.1 \quad$ Trees extraction}

Trees have different morphological characteristics, which can be used to identify them separately from remaining objects. Individual tree has two main geometrical parts (i) crown, and (ii) trunk, where crown has relatively larger area than trunk and both have near circular shape. Crown possesses wide range of variety and its shape and size changes along the length of tree. Rutzinger et al. (2010) reported that modal shapes of the tree crown could be conical, inverse conical, cylindrical and spherical. Conical, inverse conical and spherical crowns are subset of cylindrical crown. So, we assume cylindrical shape in order to incorporate all different shapes and sizes of tree crowns. Tree crowns could be detected based on compactness and area of their horizontal cross section sliced at middle of crown. Compactness (Yang et al., 2012; Yadav et al., 2016) and area of polygonal shape formed using horizontal segment can be used to distinguish the tree from other objects. Maximum $\mathrm{Z}$ value $\left(\mathrm{Z}_{\max }^{\mathrm{c}}\right.$ ) of points within each cylinder is computed. It is $\left(\mathrm{Z}_{\max }^{\mathrm{c}}\right)$ average of top 100 point from maximum in the point cloud data file arranged in decreasing order of $\mathrm{Z}$ values. In this effect of outliers is avoided. It is also assumed that there are no objects above trees and it is generally true. Vertical stretch $\left(\mathrm{H}_{\text {tree }}\right)$ of points within cylinder is computed by: $\mathrm{H}_{\text {tree }}=\mathrm{Z}_{\text {max }}^{\mathrm{c}}-$ $\mathrm{Z}_{\text {min }}^{\mathrm{c}}$ (see Figure 1 (b)). Point cloud of horizontal cross 
section (disc segment 1) of vertical length, $\Delta \mathrm{H}_{\text {crown }}=\mathrm{H}_{\text {tree }} / 10$ from cylinder is considered and it is ranged from $6 \mathrm{H}_{\text {tree }} / 10$ to $7 \mathrm{H}_{\text {tree }} / 10$ (Yadav and Chousalkar, 2017). This range is selected to consider disc segment 1 from nearly middle $\left(\mathrm{R}_{\text {crown }}^{\max }\right)$ of crown portion of tree. Compactness factor $(\mathrm{CF})$ is calculated (see Equation 1 ) for contour shape formed using disc segment 1 points.

$$
\mathrm{CF}=4 \pi \frac{\mathrm{A}}{\mathrm{p}^{2}}
$$

Where $\mathrm{A}$ and $\mathrm{p}$ are area and perimeter of contour shape, respectively. CFs for circular, square and isosceles right angle triangle contour are $1,0.79$ and 0.54 , respectively. Near circular contour shape is considered for segment points in case if concerned cylinder contains tree having crown modal shapes like conical, inverse conical, spherical, and cylindrical crowns. So, contour formed from crown segment points is approximately circular in shape (CF is more than 0.6$)$ and its area lies within $2 \pi\left(R_{\text {crown }}^{\min }\right)^{2}$ and $2 \pi\left(R_{\text {crown }}^{\max }\right)^{2}$, where, $R_{\text {crown }}^{\min }$ is minimum crown radius available in the test site. If these two conditions are satisfied by disc segment 1 then concerned cylinder might belong to tree. Again these two criteria are checked but in different horizontal cross section (disc segment 2) that belong to lower portion of cylinder at trunk level. Disc segment 2 having same centre and vertical length but small radius compare to disc segment 1(see Figure 1(b)). If contour formed by points of disc segment 2 satisfy; (i) CF more than 0.6 and, (ii) area of contour (A) lies within $2 \pi\left(R_{\text {trunk }}^{\min }\right)^{2}$ and $2 \pi\left(R_{\text {trunk }}^{\max }\right)^{2}$, where, $R_{\text {trunk }}^{\min }$ and is $R_{\text {trunk }}^{\max }$ are minimum and maximum trunk radii, respectively available in test site. Finally top to bottom cylinders fitting of different radii, i.e., $\mathrm{R}_{\text {crown }}$ and $\mathrm{R}_{\text {trunk }}$ are performed to recover complete tree structure. Height of tree, $\mathrm{H}_{\text {tree }}$ is also measured as discussed above (see Figure 1 (b)). Further extracted trees GIS database is also generated using their 2D location and height information.

\section{EXPERIMENTS}

\subsection{Mapping and Reference Data}

The proposed method is tested on the mobile LiDAR data of roadway test site acquired by StreetMapper 360 (StreetMapper 360, 2015). Test site is located along $57 \mathrm{~m}$ long narrow single lane roadway and it is composed of mainly low and high vegetation (see Figure 2).

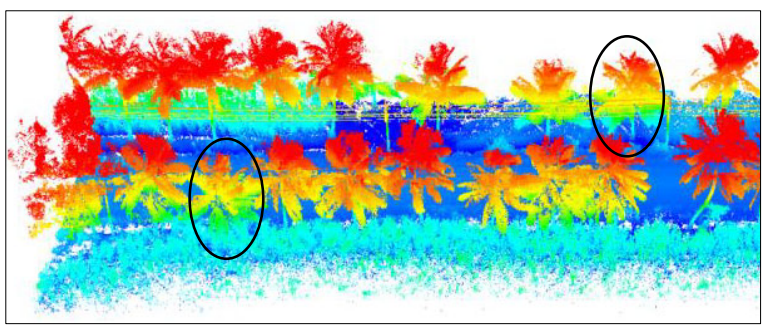

Figure 2. Point cloud view of input test site.
Road-side trees are randomly planted and most of them are connected together at their crowns. $3,106,648$ points are acquired at average 2D point density of 1240 points $/ \mathrm{m}^{2}$. Total 17 trees are present in the test site and they used as reference data.

\subsection{Results}

Proposed method is implemented on unstructured point cloud (only XYZ) of chosen test site (see Figure 2) using thresholds listed in Table 1.

\begin{tabular}{|l|c|l|l|l|}
\hline Parameters & $\mathrm{R}_{\text {crown }}^{\max }$ & $\mathrm{R}_{\text {crown }}^{\min }$ & $\mathrm{R}_{\text {trunk }}^{\max }$ & $\mathrm{R}_{\text {trunk }}^{\min }$ \\
\hline Thresholds (m) & 4 & 2.5 & 0.25 & 0.15 \\
\hline
\end{tabular}

Table 1: Thresholds of parameters used for test site.

The street trees are extracted (see Figure 3). Sequentially their GIS database (see Figure 4) is also generated using 2D location and height information (see Figure 4).

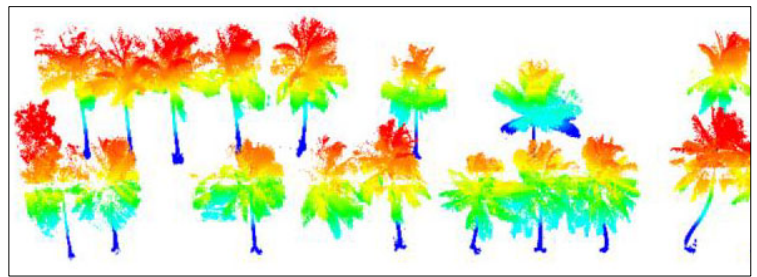

Figure 3. Point cloud view of extracted street trees.

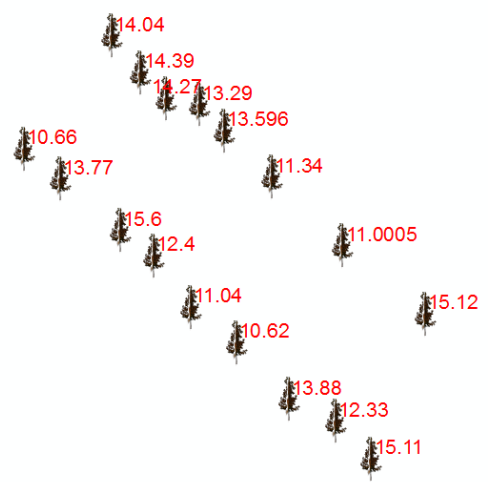

Figure 4. Map of GIS data base of extracted street trees showing locations and trees height $(\mathrm{m})$.

\subsection{Discussion}

Performance of proposed method is described using Figure $3 \& 4$ and Table 2. 15 out of 17 trees are correctly extracted. One utility pole is falsely classified as tree because it lies just below the tree. Two street trees are missing (see encircled trees in Figure 2) because their crown spreads around the trunk till the ground and occludes trunk portion. So trunk is not completely captured that why this tree do not satisfy second step of proposed method. Completeness and correctness of $88.24 \%$ and $93.75 \%$ respectively are obtained in test site. Proposed method is 
able to detect different tree species having different crown shapes, i.e. conical, inverse conical, cylindrical and spherical. Although we consider the cylindrical shape, but the proposed method is implemented on crown cross section (disc segment) at middle along its length. The cross section is nearly circular for each model shape of crown with variable radius. Therefore we consider a range of crown radius (see Table 1) to incorporate these shape models.

\begin{tabular}{|c|c|c|c|}
\hline 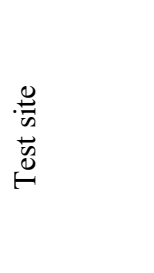 & 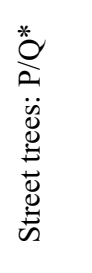 & 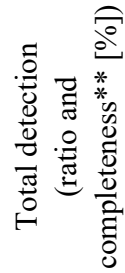 & 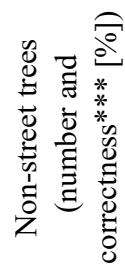 \\
\hline $\begin{array}{c}57 \mathrm{~m} \text { long } \\
\text { roadway }\end{array}$ & $15 / 17$ & $\begin{array}{c}15 / 17 \\
{[88.24]}\end{array}$ & $\begin{array}{c}01 \\
{[93.75]}\end{array}$ \\
\hline
\end{tabular}

Table 2: Number of trees detected in the test site

* $\quad \mathrm{P} / \mathrm{Q} \equiv$ Total detection/Reference value

** Completeness (\%): [Street trees detected]/ [Reference value of street trees]

*** Correctness (\%): [Street trees detected]// ([Street trees detected $]+[$ Non-street trees detected $])$

\section{CONCLUSIONS AND FUTURE WORKS}

In this study, an automatic method is proposed for extraction of tress from mobile LiDAR point cloud. Initially, data is organized into connected partially overlapping cylinders, Horizontal cross section (disc segment 1 and 2) of specific vertical length at tree crown level and trunk level, respectively are chosen from each cylinder. Compactness and area of points within disc segment 1 and 2 are computed and used as information for trees extraction. Proposed method is tested on point cloud data acquired along roadway having mainly low and high vegetation. In the point cloud data of selected test site, trees crowns are connected together and trees trunks are occluded by low vegetation unlike dataset used in literature. Completeness and correctness of $88.24 \%$ and $93.75 \%$, respectively are achieved in the selected test site. One utility pole is falsely classified as tree because it lies just below the tree. Two street trees are extracted from the point cloud because their crowns spread around the trunk till the ground and occludes trunk portion. Proposed method extracts variety of trees having different crown shape model.

Using the results obtained in test site and thorough analysis of the existing methods, it can be concluded that the proposed method is more general and it performs efficiently: (i) even in case of overlapping trees (ii) requiring no training data, (iii) no initial assumptions about the minimum separation of two adjacent trees (iv) being independent of the scanning geometry information and only need mobile LiDAR points in XYZ format.

Future works are: (i) to improve the completeness by extracting trees, which trunks are occluded by crown, (ii) modifying proposed method to make it parameter free or fully automatic, (iii) trees classification into different sub classes using crown shape as parameter.

\section{ACKNOWLEDGMENT}

The authors would like to thanks Geokno India Pvt. Ltd. for providing the StreetMapper 360 mobile LiDAR system and field assistance for capturing the point cloud data used in this study.

\section{REFERENCES}

Hyyppä, J., Hyyppä, H., Leckie, D., Gougeon, F., Yu, X., and Maltamo, M., 2008. Review of methods of smallfootprint airborne laser scanning for extracting forest inventory data in boreal forests. International Journal of Remote Sensing, 29 (5), pp. 1339-1366.

Rutzinger, M., Pratihast, A., Oude Elberink, S., and Vosselman, G., 2010. Detection and modelling of $3 \mathrm{~d}$ trees from mobile laser scanning data. In: ISPRS Archieves of Photogrammetry, Remote Sensing and Spatial Information Sciences, 38(5), pp. 521-525.

StreetMapper 360. http://www.igi.eu/streetmapper.html. (accessed on 28 Dec. 2015).

Tao, S., Wu, F., Guo, Q., Wang, Y., Li, W., Xue, B., Hu, X., Li, P., Tian, D., Li, C., and et al., 2015. Segmenting tree crowns from terrestrial and mobile LiDAR data by exploring ecological theories. ISPRS Journal of Photogrammetry and Remote Sensing, 110, pp. 66-76.

Wu, B., Yu, B., Yue, W., Shu, S., Tan, W., Hu, C., Huang, Y., Wu, J., and Liu, H., 2013. A voxel-based method for automated identification and morphological parameters estimation of individual street trees from Mobile Laser Scanning data. Remote Sensing, 5 (2), pp. 584-611.

Yadav, M., Chousalkar, C.G., 2017. Extraction of power lines using mobile LiDAR data of roadway environment. Remote Sensing Applications: Society and Environment, 8, pp. 258-265.

Yadav, M., Goel, S., Singh, A. K., and Lohani, B., 2014. Developing Basic Design and Mathematical Framework for a Mobile Mapping System—a Case Study Using Available 
Sensors, Journal of Indian Society of Remote Sensing, 42(2), pp. 301-310.

Yadav, M., Husain, A., Singh, A. K., and Lohani, B., 2015a. Pole-shaped object detection using mobile LiDAR data in rural road environments. In: ISPRS Annals of the Photogrammetry, Remote Sensing and Spatial Information Sciences, Vol. II-3/W5, pp. 11-16.

Yadav, M., Lohani, B., Singh, A. K., Husain, A., 2016. Identification of pole-like structures from mobile LiDAR data of complex road environment. International Journal of Remote Sensing, 37(20), pp. 4748-4777.

Yadav, M., Singh, A. K., Lohani, B., 2017. Extraction of road surface from mobile LiDAR data of complex road environment. International Journal of Remote Sensing, 38(16), pp. 4645-4672.

Yadav, M., Singh, A. K., 2018. Rural Road Surface Extraction Using Mobile LiDAR Point Cloud Data. Journal of Indian Society of Remote Sensing, 46(4), pp. 531-538.

Yang, B., Wei, Z., Li, Q., and Li, J., 2012. Automated extraction of street-scene objects from mobile LiDAR point clouds. International Journal of Remote Sensing, 33(8), pp. 5839-5861

Yu, Y., Li, J., Guan, H., Zai, D., and Wang, C., 2014. Automated extraction of $3 \mathrm{~d}$ trees from mobile LiDAR point clouds, In: ISPRS Archieves of Photogrammetry, Remote Sensing and Spatial Information Sciences, 40(5), pp. 629632. 